\title{
The Periodontal Disease Index (PDI)
}

\author{
BY SIGURD P. RAMFJORD*
}

THE PERIODONTAL Disease Index is a clinician's modification of Russell's PI index for epidemiological surveys of periodontal disease. The PDI index is primarily concerned with an accurate assessment of the periodontal status of the individual person. Emphasis is placed on recording of the attachment level of the periodontal tissues relative to the C-E junction. Such accurate measurable assessments are essential for longitudinal studies of periodontal disease and as a scientific basis for clinical trials in Periodontology.

\section{Objectives of the PDI Index}

The following objectives were incorporated into the design of the index:

1. To assess prevalence and severity of gingivitis and periodontitis within the individual dentitions and in population groups.

2. To provide an accurate basis for incidence and longitudinal studies of periodontal disease.

3. To provide a meaningful basis for estimate of need for periodontal therapy in selected population groups.

4. To provide accurate recordings for clinical trials of preventive and therapeutic procedures in periodontics.

5. To provide measurable reference data for assessment of correlations with factors of potential significance in the etiology of periodontal disease.

\section{Scoring Methods}

In the 20 minutes allowed me for this presentation I will mainly confine my remarks to clarification of the scoring meth-

* The University of Michigan School of Dentistry, Ann Arbor, Michigan.

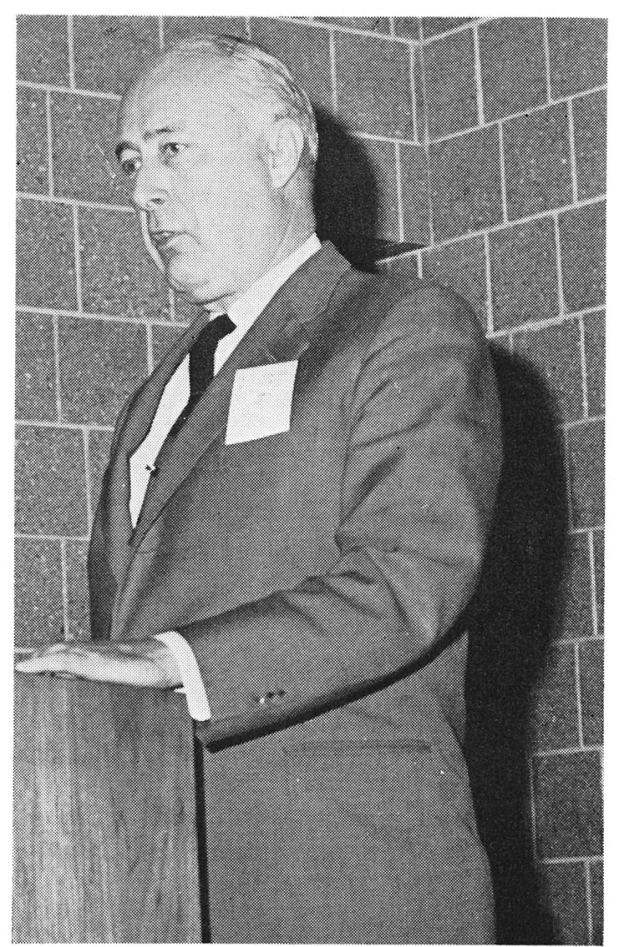

S. Ramfjord presenting.

ods. Accuracy and reproducibility of scoring becomes increasingly more dependent on the training of the person who is going to do the scoring as demands for accuracy are increased, and the merits of this index should not be evaluated until a person has had considerable training and experience in the use of the system. Assessment of degree of periodontal disease includes a subjective assessment of color, form, density, and bleeding tendency of the gingival tissues. But by far the most important feature of the PDI index is measurement of the level of the periodontal attachment related to the C-E junction of the teeth. The location of the reference marks needed for the measurements however is based on touch 
rather than on vision, which means that the investigator has to be trained to locate the C-E junction and the bottom of the epithelial attachment accurately by touch. If these reference marks are covered over by calculus they have to be exposed. In a few instances the original cementum-enamel junction may have been lost due to excessive abrasion or dental restorative procedures. However, in the great majority of individuals the cementum-enamel junction can be located by a well trained investigator. It should be emphasized that this method is entirely unreliable in the hands of an untrained investigator.

Maximal accuracy also depends on standardized optimal lighting and standardized thickness of the measuring probes. Only 6 selected teeth are scored for assessment of the periodontal status of the mouth; however, for short term clinical trials and where a limited number of patients are available, one may concern all of the teeth in the mouth. The 6 selected teeth are: tooth \#3 (maxillary right first molar), tooth \#9 (maxillary left central incisor), tooth \#12 (maxillary left first bicuspid), tooth \#19 (mandibular left first molar), tooth \#25 (mandibular right central incisor) and tooth \#28 (mandibular right first bicuspid). It has been shown by Jamison and in a number of published and unpublished studies from our own institution that these 6 teeth provide basis for a surprisingly accurate assessment of the total periodontal status of the individual as expressed in scoring of all of the teeth.

The gingival status is scored first. The method and the assigned value represents essentially a combination of the PMA and the PI index, with the following definitions of criteria:

$0=$ absence of signs of inflammation

$1=$ mild to moderate inflammatory gingival changes, not extending around the tooth

$2=$ mild to moderately severe gingivitis extending all around the tooth

$3=$ severe gingivitis characterized by marked redness, swelling, tendency to bleed and ulceration.
Since all these criteria are based on subjective values and examiners judgment $I$ would like to describe in detail the basis for such judgment. The gingivae around the teeth to be scored are first dried superficially by gently touching with absorbing cotton. Changes in color are evaluated by observing the color of the gingiva around the tooth to be scored and comparing the color corresponding to the buccal, lingual and interproximal surfaces with each other, as well as comparing it with the color of the gingiva around adjacent teeth. We pay more attention to the uniformity or lack of uniformity of color than to color shades or hues, since we assume that the physiologic factors which determine different color shades of gingiva are constant around the tooth and for the adjacent teeth. Color changes are usually towards redness but there may also be changes towards a bluish or purplish hue.

Change in form is initially a blunting or rounding of the margin of the gingiva and thickening of papilla; however, gingivitis is never scored on the basis of a slight contour change alone since this may not necessarily indicate the presence of disease. Very little significance is given to the presence or absence of stippling since this does not necessarily relate to presence or absence of gingival inflammation.

Change of consistency or density is detected by applying gentle pressure with the side of the periodontal probe against the gingiva to determine if there is a soft or spongy consistency. If there is a clearly detectable color change indicating gingivitis, the consistency is not tested. Any minor change either in contour, stippling or consistency alone is not considered to be a definite manifestation of gingivitis.

The score of 3 is based on evidence of ulceration of the gingiva with bleeding, if the gingiva is touched gently with the side of a periodontal probe, or if there is severe redness and marked change in contour. The score of 3 is given even if these changes do not extend all around the tooth. 
The next step in the scoring procedure is recording of crevice depth related to the $\mathrm{C}-\mathrm{E}$ junction. For this purpose we use a University of Michigan \# 0 probe, made by the Premier Mfg. Co. in Philadelphia. We have encountered great problems in standardizing the manufacturing of this probe and it still is not the instrument that we would like to have. We have had problems with variation in thickness, with inaccurate placement of the reference marks, variations in clarity of definition of the reference marks, and variations in angulations. Besides the obvious errors that can result from variation in placement of the reference marks, a slight variation in thickness may influence the results when populations with fairly normal and dense gingival tissues are scored. A thin probe under these circumstances will penetrate deeper than a thicker probe and consequently give higher scores.

The probe should be held with a light grasp similar to the manner of holding a pencil and balanced well in the hand so it can be moved and directed by very small forces. The end of the probe should be placed against the enamel surface coronally to the margin of the gingiva so that the angle formed by the working end of the probe and the long axis of the crown of the tooth is approximately $45^{\circ}$. A minimal force should be used to pass the probe in apical direction maintaining contact with the tooth. The angle between the probe and the tooth may have to be decreased slightly when the probe touches the gingiva to avoid pressure on the gingiva when the probe is inserted in the gingival crevice.

Since the surfaces of the enamel and the cementum have different inclines, the change in direction of the movement of the point of the probe is detectable when it moves from the enamel to the cementum. The texture or surface characteristics are also different since the cementum is distinctly rougher than enamel. A very important point is to use a light grip on the probe otherwise the keen sense of touch in the fingertips becomes impaired. The probe should always be pointed towards the apex of the tooth or the central axis of multirooted teeth. After the distance from the free gingival margin to the cementum enamel junction has been measured, an attempt should be made to move the probe along the cemental surface. This of course can be achieved only if there has been loss of periodontal attachment. If calculus covers the cementum enamel junction it has to be removed before the $\mathrm{C}-\mathrm{E}$ junction can be localized. Occasionally it is also necessary to remove heavy deposits of supragingival calculus to gain access to the gingival crevice. The University of Michigan \# 0 probe is graduated at 3,6 , and $8 \mathrm{~mm}$. from the end, making it necessary to estimate intervening measurements. In our experience, reproducibility is better and eye strain less following proper training with this probe than with probes that have marks for every mm.

All measurements are rounded to the nearest $\mathrm{mm}$.; except that anything close to $1 / 2$ a $\mathrm{mm}$. is always rounded to the lower whole number. It has been found in our combined histometric and clinical studies that there is a tendency with a thin probe to record a slightly greater depth than to the coronal level of the connective tissue attachment to teeth. Thus we do not record for instance $1 \mathrm{~mm}$. of pocket depth below the C-E junction and indicate loss of attachment unless we are sure that the probing extends definitely more than $1 / 2$ $\mathrm{mm}$. from the C-E junction. By assigning all the doubtful measurements to the lower score the reproducibility is much greater than if a more accurate determination of $1 / 2 \mathrm{~mm}$. were attempted.

We have during the last two years modified the PDI index so we are now scoring only buccal and mesial measurements. It has been found both by Jamison and Ash in separate extensive analysis, although yet unpublished, that there is no significant loss of accuracy in the PDI index from omitting the lingual and distal scores. Omit- 


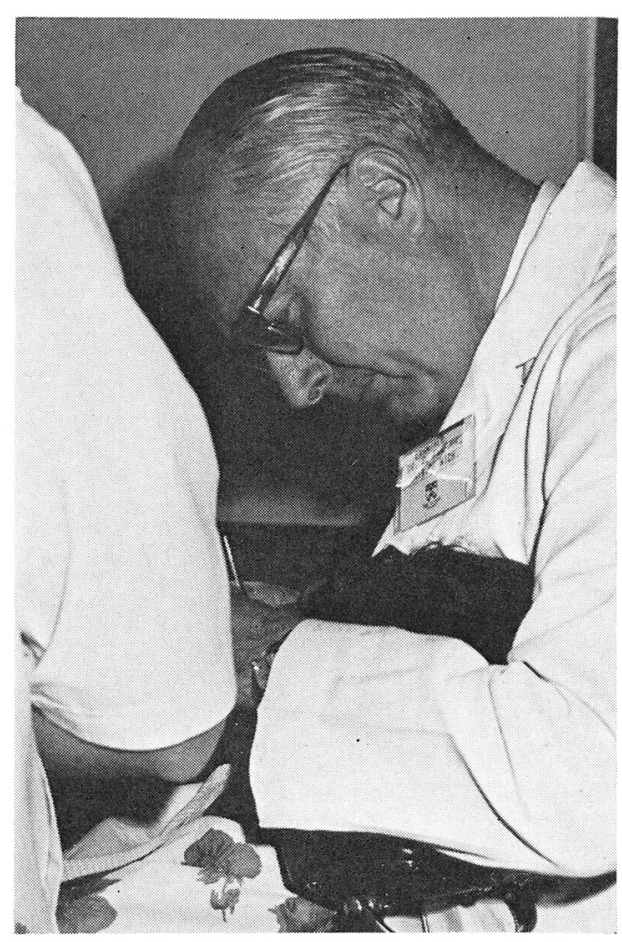

S. Ramfjord examining.

ting the lingual and distal scores has made it much easier to achieve reproducibility in buccal and mesial scoring than when we included distal and lingual scoring. However, in some clinical trials where there are a limited number of cases available and the investigators are well trained, we may still use all 4 measurements for each tooth.

The crevicular measurements are recorded in the following manner: The distance from the free gingival margin to the cementum enamel junction and the distance from the free gingival margin to the bottom of the gingival crevice or pocket is measured for the buccal and mesial aspect of each tooth examined. The buccal measurements should be made at the middle of the buccal surfaces. The mesial measuring should be made at the buccal aspect of the interproximal contact area with the probe touching both teeth if there is a neighbor tooth present and the probe pointing in the direction of the long axis of the tooth to be scored. Follow this routine for recording:

A. If the gingival margin is on enamel, measure from gum margin to cementumenamel junction and record the measurement. If the epithelial attachment is on the crown and the cementum enamel junction cannot be felt by the probe, record the depth of the gingival crevice on the crown. Then record the distance from the gingival margin to the bottom of the pocket if the probe can be moved apically to the cementum-enamel junction without resistance or pain. The distance from the cementum-enamel junction to the bottom of the pocket can then be found by subtracting the first from the second measurement.

B. If the gingival margin is on cementum, record the distance from the cementum-enamel junction to the gingival margin as a minus value. Then record the distance from the cementum-enamel junction to the bottom of the gingival crevice as a plus value. Both loss of attachment and actual crevice depth can easily be assessed from these scores.

\section{Scoring of Calculus}

Calculus is scored on the basis of the following criteria:

$0=$ absence of calculus

1 :- supragingival calculus extending only slightly below the free gingival margin (not more than $1 \mathrm{~mm}$.)

$2=$ moderate amount of supra and subgingival calculus or subgingival calculus alone

$3=$ an abundance of supra and subgingival calculus.

Subgingival calculus apparently is much more important in the pathogenesis of periodontitis than supragingival calculus and consequently it has been given a higher score than supragingival calculus. Subgingival calculus is located with a \#17 probe if there is uncertainty when probing with the University of Michigan \#0 probe. 


\section{Scoring of Plaque}

Scoring of plaque is done after staining with Bismarck brown solution. Bismarck brown solution is placed in a dappen dish, and two Richmond cotton pellets \# 0 are placed in the dish until they appear completely saturated with the solution. The patient is asked to swallow and one saturated pellet is removed with a cotton plier and touched gently onto the lingual and buccal surfaces of the mandibular teeth. The second saturated pellet is touched onto the palatal and buccal surfaces of the maxillary teeth. The occlusal surfaces are also rubbed with the pellet so the disclosing solution flows over all the surfaces of the teeth. The patient is then instructed to spit in the cuspidor and rinse his mouth thoroughly twice. The scoring is now done according to the following criteria:

$0=$ no plaque present

$1=$ plaque present on some but not on all interproximal buccal and linqual surfaces of the tooth

$2=$ plaque present on all interproximal, buccal and lingual surfaces, but covering less than one half of these surfaces

$3=$ plaque extending over all interproximal, buccal and lingual surfaces, and covering more than one half of these surfaces.

Only full erupted teeth should be scored and missing teeth should not be substituted.

The scoring of the calculus and plaque does not constitute a part of the PDI index, but since an extremely high correlation has been established between periodontal disease and the presence of plaque and calculus we usually include plaque and calculus score in total assessment of periodontal status.

\section{Computation of Indices from \\ Recorded Data}

The recorded data can be utilized as a basis for individual indices for gingivitis, for total periodontal disease (PDI), for calculus and for plaque or for calculus and plaque combination. The gingivitis index is derived at by adding the scores for gingivitis for all of the examined teeth and then divide the sum by the number of teeth examined. The PDI index for the patient is the average of the PDI index for the examined teeth, which is based on the following tabulation: If the gingival crevice in none of the measured areas extended apically to the cementum-enamel junction the recorded score for gingivitis is the PDI score for that tooth. If the gingival crevice in any of the two measured areas extended apically to the cementum-enamel junction but not more than $3 \mathrm{~mm}$. (including $3 \mathrm{~mm}$. in any area) the tooth is assigned a PDI score of 4 . The score for gingivitis then is disregarded in the PDI score for that tooth. If the gingival crevice in any of the two recorded areas of the tooth extends apically to from 3 to $6 \mathrm{~mm}$. (including $6 \mathrm{~mm}$.) in relation to the cementum-enamel junction the tooth is assigned the PDI score of 5 (again the gingivitis score is disregarded). Whenever the gingival crevice extends more than $6 \mathrm{~mm}$. apically to the cementumenamel junction in any of the measured areas of the tooth, the score of 6 is assigned as the PDI score for that tooth (again disregarding the gingivitis score).

The total PDI score for the patient is then computed by addition of the individual scores of the teeth and the sum is divided by the number of scored teeth. Whether or not the periodontal support has been lost because of periodontitis or atrophy is not considered in the PDI index.

If some of the teeth which were scheduled to be examined were missing or unerupted so they could not be examined, then individual scores for each of the examined teeth should be added and divided by the number of teeth examined and there should be no substitution for absent teeth.

Indices for calculus and plaque are tabulated in the same manner as the PDI index. The scores for calculus for each individual tooth examined are added and the sum divided by the number of teeth examined to yield the person's index for calculus. 
The scores for plaque for each tooth examined also are added and divided by the number of examined teeth, thus the index of plaque hygiene is tabulated.

The mean of the calculus and the plaque index will give an expression for the combined potential irritation from these two main etiological factors related to lack of oral hygiene or an "irritation index" as indicated by O'Leary.

\section{Examiner Scoring Deviation and Calibration}

The observers ability to assess repeatedly in a reproducible way the scoring criteria in actual clinical situations have to be determined by errors tests prior to the study. The examiner should also be retested during a study that extends over any prolonged period of time. Control of accurate adherence to the scoring criteria and reproducibility is especially important for clinical trials and longitudinal studies.

One way of assessing scoring deviation is as follows: After the investigator has learned to use the index to the point that scoring becomes automatic and he can call off the figures without any hesitation, the following scoring test can be applied. Select 7 adult patients at random and score each patient 5 times (not consecutively) for gingivitis, crevice depth, calculus and plaque. The first and second, second and third, third and fourth, fourth and fifth, and first and fifth scores for the individual teeth of each patient can be compared for gingivitis, periodontal crevice depth, calculus and plaque. No change is recorded as 0 , increases as +1 or +2 or decreases as -1 or -2 in individual scores for the examined teeth based on scoring units. In one recent such test administered to a dental hygienist doing a toothbrush study her average deviation for plaque score was $\pm 0.062 ; \pm 0.018$ for calculus; \pm 0.033 for gingivitis; \pm 0.000 for periodontal scores and for crevice depth \pm 0.004 . A greater number of such tests have been directed by Dr. Ash and in general the accuracy figures are very high for crevice depth determinations, slightly lower for calculus and plaque and lowest for gingivitis. However, the reproducibility figures for gingivitis still are fairly good as can be seen from the data I just quoted to you.

Another method is also used to determine variability in scoring for a single test calibration and for tests before, during, and after a study has been completed. Usually some change will take place, the amount will depend upon initial training. Initial training should include a wide range of subjects with a sufficient range in value of scores to prevent presentation of a number of difficult scoring problems.

In a test scoring situation using 7 subjects and 5 observations the usual procedure is to determine the pooled estimate of variance of the measurement errors (Table 1). The variances from two tests, one at the beginning and another at the end of the study, can be tested on the basis of an $F$ ratio. It is obvious that a knowledge of variation and scoring error should be known throughout a study if comparisons between studies or more than one observer is to be used.

Besides the individual examiners error one of course has to consider calibration for interexaminer errors. It is not within the scope of this paper to indicate how such tests should be organized; however, it has to be emphasized that calibration tests are of essential importance in all forms of clinical studies.

\section{Recommended Use of the PDI Index and Associated Data}

Periodontal indices are sometimes classified as irreversible and reversible indices. An irreversible index assesses the damage caused by a disease rather than the disease itself. A classical example of such an index is the DMF index for dental caries. The PDI index has often been referred to as an irreversible index since it measures the destruction caused by periodontal disease and 
TABLe 1

Test

\begin{tabular}{lccccccccccc}
\hline \multirow{2}{*}{ Patient } & 1 & 2 & 3 & 4 & 5 & $\Delta^{\prime} s$ & $N$ & $\Sigma_{x}$ & $\Sigma_{x^{2}}$ & $\Sigma(x-\bar{x})^{2}$ & $d f$ \\
\hline 1 & 1 & 2 & 1 & 2 & 1 & 4 & 5 & 7 & 11 & 1.2 & 4 \\
2 & 2 & 2 & 2 & 2 & 2 & 0 & 5 & 10 & 20 & 0.0 & 4 \\
3 & 3 & 2 & 3 & 2 & 3 & 4 & 5 & 13 & 35 & 1.2 & 4 \\
4 & 3 & 2 & 2 & 2 & 2 & 1 & 5 & 11 & 25 & 0.8 & 4 \\
5 & 2 & 2 & 2 & 1 & 2 & 2 & 5 & 9 & 17 & 0.8 & 4 \\
6 & 1 & 1 & 0 & 1 & 0 & 3 & 5 & 3 & 3 & 1.2 & 4 \\
7 & 2 & 1 & 2 & 2 & 2 & 2 & 5 & 9 & 17 & 0.8 & 6.0 \\
\end{tabular}

Difference between

successive steps

$$
=\frac{16}{28}=.571
$$

$$
\begin{aligned}
& \mathrm{s}_{\mathrm{e}}^{2}=\frac{\Sigma \Sigma(\mathrm{x}-\overline{\mathrm{x}})^{2}}{\mathrm{df}}=\frac{6.0}{2.8} \\
& \mathrm{~s}_{\mathrm{e}}^{2}=.2143 \\
& \mathrm{~s}_{\mathrm{e}}=.463
\end{aligned}
$$

it has been assumed that the destruction caused by periodontal disease was irreversible.

A reversible index indicates the possibility: for the score to return to zero, as a result of cure of the disease that was indexed. In other words a reversible index assesses an active disease which may be completely cured while irreversible indices assess permanent damage caused by a disease. It has been implied that for clinical trials of treatment for periodontal disease one would need reversible indices.

This is an entirely meaningless characterization of indices because if the index reports the status of health or disease accurately, reversals or lack of reversals will appear when the data are compared regardless whether the tag of reversible or irreversible has been placed on the index.

It is however, extremely important to know how the data obtained from the examination, scoring and indexing can best be utilized and applied to problem solving in clinical trials. The first objective of the PDI index was to find a numerical expression of the status of periodontal health or disease in a given individual. Then this information could be used for assessment of the periodontal status of population groups following proper sampling. Looked upon from this standpoint the PDI index is essentially a clinician's modification of the Russell PI index and for this purpose it behaves essentially the same way as the PI index. The similarity in behavior of these two indices has been documented by comparison of data from Greene's and my study in India and from Jamison's work.

Being a clinician rather than an epidemiologist I mainly like to discuss with you the clinical significance of the PDI indexing. It serves to map the clinical behavior of periodontal disease in any given individual as well as in population groups. Thus it may be used as a guide in assessing need for treatment and for evaluation of results of treatment. More specifically it can be stated that data accumulated associated with PDI indexing are useful for the following purposes:

1. It records gingivitis for the selected teeth and provides an average value for gingivitis in the entire mouth. It is generally accepted that simple gingivitis is a reversible disease. Cure of gingivitis or fluctuation in severity of gingivitis both for 
individual teeth and for the entire mouth can be assessed from the gingivitis scores. Other indices may of course provide such data equally well or better.

2. It provides the data needed for assessment of prevalence of both gingivitis and periodontitis separately, besides measuring the severity of these conditions. It may be of great clinical significance to know at what age periodontitis (periodontal destruction) starts.

3. It provides meaningful data for assessment of the total need of periodontal treatment for individuals and for populations.

4. It establishes an accurate record of the level of periodontal support for the selected teeth at the time of the indexing. It was found in a recent study that this part of the index is not necessarily as irreversible as it has been claimed since periodontal support may be regained as a result of treatment. The PDI index or the recorded data from such indexing can pinpoint such gain or loss of support.

If the recorded data are going to be used for clinical trials, separate analysis should be done for the impact of the clinical trial upon gingivitis, and for the impact of the same clinical trial upon crevice depth and level of periodontal attachment around the teeth. As an example it can be mentioned that data from a recent clinical trial involving periodontal therapy showed some gain of periodontal support in the interproximal areas following curettage, while a surgical lowering of buccal and lingual attachment was followed by gradual return to the presurgery level.

5. Clinical research concerning pathogenesis of periodontal disease with experimentally induced irritation requires the greatest possible accuracy in accumulation of measurable data. Information from the PDI indexing provides measurable data re- garding pocket formation related to loss of attachment.

6. Testing of methods and devices for oral hygiene and preventive periodontics should include accurate information both regarding the impact on gingivitis and effectiveness in prevention of periodontal destruction. Data from the PDI indexing will provide basis for separate analysis of these two factors.

I want to emphasize that the data accumulated during the PDI indexing lend themselves very well for separate analysis of gingivitis and level of periodontal attachment; thus provides a great amount of versatility beyond the information that can be obtained from a simple PDI indexing. The data obtained in millimeters are quantitative and suitable for the types of mathematical manipulations necessary for many forms of statistical analysis. Analysis of the data should be directed towards the type of information that is desired.

\section{Final Remarks}

By now I am sure that you all have concluded that as usual I have gone way overboard and in my enthusiasm have tried to sell you a "perfect tool" for clinical studies. You know of course that there is no such thing as a perfect tool in this field where subjective judgments play havoc with mathematical values. Furthermore, we are fishing with a large masked net which does not allow us to catch small fishes. There is an obvious need for refinements in devices and techniques. We have for example refined our plaque scores for toothbrush studies in populations with relatively good oral hygiene to get a more accurate assessment of small variations. We are modifying our data analysis to suit the various problems that we are investigating. We have not been able to come up with the type of periodontal probe that we would like to have, and we are steadily experimenting with methods for more expedient training of examiners. Beyond all we are looking for better ways to catch more fish!! 


\section{REFERENCES}

1. Ackerman, M. Anne: A clinical study of the Dominion electric toothbrush. Typed thesis. Univ, of Mich. School of Dentistry, Ann Arbor, 1965. p. 86.

2. Greene, J. C.: Periodontal disease in India: Report of an epidemiological study. J. dent. Res., 39:302312, 1960.

3. Jamison, Homer: Prevalence and severity of periodontal disease in a sample of a population. Typed thesis. Univ. of Mich. School of Public Health, Ann Arbor, 1960.153 p.

4. Ramfjord, S. P.: Indices for prevalence and inci- dence of periodontal disease. J. Periodont., 30:51-59, 1959.

5. Ramfjord, S. P., Nissle, R. R., Shick, R. A. and Cooper, H.: Subgingival curettage versus surgical elimination of periodontal pockets. J. Periodont. (In press).

6. Russell, A. L.: A system of classification and scoring for prevalence surveys of periodontal disease. J. dent. Res., 35:350-359, 1956.

7. Smith, W. A. and Ash, M. M., Jr.: A clinical evaluation of an electric toothbrush. J. Periodont., 35: 127-136, 1964.

\section{The Gingival Index, the Plaque Index and the Retention Index Systems

\author{
BY HARALD LÖE
}

\section{THE GINGIVAL INDEX (GI)}

The main purpose of creating the Gingival Index system was to introduce a system for the assessment of the gingival condition which clearly distinguished between the quality of the gingiva (the severity of the lesion) and the location (quantity) as related to the four (buccal, mesial, distal, lingual) areas which make up the total circumference of the marginal gingiva (Löe and Silness, 1963). At the time the GI was taken into use the existing index systems, the PMA index (Massler and Schour; 1949) with later modifications, the Periodontal Index (Russell, 1957) and the Periodontal Disease Index (Ramfjord, 1959), did not fulfill this requirement.

The Gingival Index does not consider periodontal pocket depth, degrees of bone loss or any other quantitative change of the periodontium. The criteria are entirely confined to qualitative changes in the gingival soft tissue.

Department of Periodontology, The Royal Dental College, Aarhus, Denmark.
CRITERIA FOR THE GINGIVAL INDEX SYSTEM

$0=$ Normal gingiva

$1=$ Mild inflammation - slight change in color, slight oedema. No bleeding on probing

$2=$ Moderate inflammation-redness, oedema and glazing. Bleeding on probing

$3=$ Severe inflammation - marked redness and oedema. Ulceration. Tendency to spontaneous bleeding.

Each of the four gingival areas of the tooth is given a score from 0 to 3 ; this is the GI for the area. The scores from the four areas of the tooth may be added and divided by four to give the GI for the tooth. The scores for individual teeth (incisors, premolars and molars) may be grouped to designate the GI for the group of teeth. Finally, by adding the indices for the teeth and dividing by the total number of teeth examined, the GI for the individual is obtained. The index for the subject is thus an average score for the areas examined.

$\mathrm{GI}=0$ is given to the gingiva the color of which is pale pink to pink. The 